\title{
Market basket survey shows elevated levels of As in South Central US processed rice compared to California: Consequences for human dietary exposure
}

Williams PN, Raab A, Feldmann J \& Meharg $\mathbf{A A}^{1^{*}}$

Supporting Information

$$
\begin{aligned}
& \text { Pages }=24 \\
& \text { Figures }=2 \\
& \text { Tables }=17
\end{aligned}
$$


S3-4 Method

S4 Results \& Discussion

S5 Table S1. Summary of Arsenic levels in U.S. rice

S6 Table S2. Summary of Selenium levels in U.S. rice

S7 Table S3. Summary of Copper levels in U.S. rice

S8 Table S4. Summary of Manganese levels in U.S. rice

S9 Table S5. Summary of Cobalt levels in U.S. rice

S10 Table S6. Summary of Zinc levels in U.S. rice

S11 Table S7. US Rice Brands.

S12 Table S8. Summary of the element levels in polished/unpolished US Rice.

S13 Table S9. Analysis of variance for transition metals.

S14 Table S10. Analysis of variance for arsenic and selenium.

S15 Fig S1. Interaction plots for polished and unpolished rice from either Californian or South Central US rice.

S16 Table S11. Background levels of Se, As and Mn in soils from non-agricultural soils from rice producing regions of the US.

S17 Table S12. Summary of total and water soluble As in soils from the state of Mississippi.

S18 Table S13. Summary of As and Se levels in stream sediments from rice producing counties in the US.

S19 Table S14. Summary of As levels in potable groundwaters, by State.

S20 Table S15. Quality Control.

S21 Table S16. Duplicate analysis of samples

S22 Table S17. Composition and concentration of mulielement standard.

S23 Fig S2. (a) Regression of As grain level vs. PCA Axis 2.

(b) Loading plot of As, Se, Cu, Mn, Co, Zn.

S24 References 


\section{Method:}

Chemicals. Aristar grade reagents were used exclusively throughout the analysis. Nitric Acid was obtained from VWR International. $1000 \mathrm{mg} / \mathrm{L}$ standards of Co, As, $\mathrm{Se}, \mathrm{Cu}, \mathrm{Zn}, \mathrm{Mn}$ and In were obtained from Merck.

Statistics. General Linear Modelling (GLM) and the Mann Whitney test were conducted using Minitab v.14 (State College, PA). Data used in the GLM were ranked prior to analyses to normalise distribution.

Sample Preparation. Raw rice samples were powdered using a marble "Pulverisette" Ball-mill (Fritsch, Germany), then oven dried at $90^{\circ} \mathrm{C}$ for 48 hours. Approximately $0.2 \mathrm{~g}$ of rice flour/powder was weighed out into a $50 \mathrm{ml}$ polypropylene digest tube and $2 \mathrm{mls}$ of conc. nitric acid were added; the mixture was left to steep overnight. Samples were digested in a microwave oven (CEM Mars 5, CEM Corp, Matthews, NC). The temperature was gently raised, first to $55^{\circ} \mathrm{C}$ (and held for ten minutes) then to $75^{\circ} \mathrm{C}$ (and held for ten minutes). Finally the digest was taken up to $95{ }^{\circ} \mathrm{C}$ and maintained for 30 minutes. Samples were cooled to room

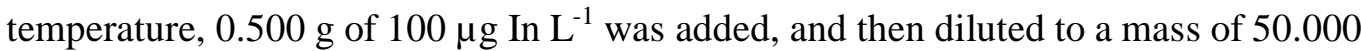
$\mathrm{g}$ with Ultrapure deionized water (18.2 M $\Omega$ ) obtained from a Milli-Q system (Millipore). Quality controls of CRM, spikes and blanks were run with each digest set of $\sim 45$ samples.

Total Element Detection. An Agilent ${ }^{\mathrm{TM}}$ 7500c (Agilent Technologies, Toyoko, Japan) octopole reaction system (ORS)-ICP-MS, with a Meinhard nebulizer was used to measure the elements $\mathrm{Ti}(\mathrm{m} / \mathrm{z} 49), \mathrm{Mn}(\mathrm{m} / \mathrm{z} 55), \mathrm{Co}(\mathrm{m} / \mathrm{z} 59), \mathrm{Cu}(\mathrm{m} / \mathrm{z} 63), \mathrm{Zn}$ 
$(\mathrm{m} / \mathrm{z}$ 64, 66, 67, 68), As $(\mathrm{m} / \mathrm{z}, 75)$, Se $(\mathrm{m} / \mathrm{z} 77,78,82)$ and $\operatorname{In}(\mathrm{m} / \mathrm{z} 115)$. Hydrogen was employed as the reaction gas for ORS, set at a flow rate of $1.8 \mathrm{~mL} / \mathrm{min}$. The following $m / z, 77,78,82$ were measured in order to identify polyatomic $\mathrm{Ar}^{40} \mathrm{Cl}^{35}$ interferences on $m / z$ 75. Corrections for interference from $\mathrm{Ar}^{40} \mathrm{Cl}^{35}$ were not found to be necessary. Samples were randomised prior to analysis. Standards were run after every set of twenty samples. Every tenth sample was digested and analysed in duplicate (Table S16). NIST Certified Reference Material 1568a Arkansas long grain rice flour was used to validate the analysis. All sample vials were cleaned then soaked in $10 \% \mathrm{v} / \mathrm{v}$ nitric acid, for a minimum of 12 hours then washed with deionised water and air dried, prior to use. Concentrations were determined using five point calibrations (Table S17.) calculated from a multielement standard, and adjusted with the blanks.

\section{Results and Discussion:}

\section{Total Level of Transition element metals in American Rice Grain.}

For the transition element metals of manganese, cobalt, copper and zinc (Fig 1. and Table 1.) no significant difference in grain level was observed between rice from California and the South Central (GLM: Mn p =0.777, Co p $=0.509, \mathrm{Cu} p=0.317$. Mann Whitney; Zn p = 0.6532). However significant differences in levels were observed between polished and unpolished rice (GLM: Mn $\mathrm{p}<0.001$, Co $\mathrm{p}<0.001$, $\mathrm{Cu}<0.001$. Mann Whitney; $\mathrm{Zn} \mathrm{p}<0.001)$. This indicates that the nutritional qualities of rice are reduced upon polishing. The average levels of $\mathrm{Cu}, \mathrm{Mn}, \mathrm{Co}$, and $\mathrm{Zn}$ in the US rice grain were higher than the tested Californian purchased Bangladesh market rice. 


\begin{tabular}{|c|c|c|c|c|c|c|c|}
\hline & \multirow[t]{2}{*}{ Origin } & \multirow[t]{2}{*}{ Brand } & \multicolumn{4}{|c|}{$\begin{array}{c}\text { Total As } \\
\text { concentration, } \mu \mathrm{g} \mathrm{g}^{-1}\end{array}$} & \multirow[b]{2}{*}{$\mathrm{n}$} \\
\hline & & & $\min$ & $-\max$ & mean & median & \\
\hline \multirow[t]{3}{*}{ Overall } & California & & 0.10 & -0.30 & 0.17 & 0.16 & 27 \\
\hline & South Central & & 0.15 & -0.66 & 0.30 & 0.27 & 107 \\
\hline & & Average & 0.10 & -0.30 & 0.17 & 0.16 & 27 \\
\hline \multirow[t]{29}{*}{ Region/State } & California & A & 0.10 & -0.25 & 0.15 & 0.15 & 15 \\
\hline & & B & 0.20 & -0.30 & 0.26 & 0.27 & 6 \\
\hline & & $\mathrm{C}$ & 0.11 & -0.12 & 0.12 & 0.12 & 2 \\
\hline & & $\mathrm{D}$ & 0.12 & -0.15 & 0.13 & 0.13 & 2 \\
\hline & & E & 0.21 & -0.21 & 0.21 & 0.21 & 1 \\
\hline & & $\mathrm{F}$ & 0.15 & -0.15 & 0.15 & 0.15 & 1 \\
\hline & & Average & 0.19 & -0.66 & 0.31 & 0.26 & 51 \\
\hline & Mississippi & G & 0.23 & -0.51 & 0.34 & 0.27 & 26 \\
\hline & Delta & $\mathrm{H}$ & 0.19 & -0.48 & 0.25 & 0.24 & 20 \\
\hline & & I & 0.28 & -0.36 & 0.32 & 0.32 & 3 \\
\hline & & $\mathrm{J}$ & 0.66 & -0.66 & 0.66 & 0.66 & 1 \\
\hline & & $\mathrm{K}$ & 0.28 & -0.28 & 0.28 & 0.28 & 1 \\
\hline & & Average & 0.15 & -0.57 & 0.34 & 0.34 & 14 \\
\hline & Texas & $\mathrm{L}$ & 0.15 & -0.57 & 0.33 & 0.32 & 13 \\
\hline & & M & 0.50 & -0.50 & 0.50 & 0.50 & 1 \\
\hline & & Average & 0.17 & -0.42 & 0.27 & 0.27 & 42 \\
\hline & South Central & $\mathrm{N}$ & 0.18 & -0.41 & 0.28 & 0.27 & 19 \\
\hline & (Unknown State) & $\mathrm{O}$ & 0.17 & -0.42 & 0.26 & 0.24 & 12 \\
\hline & & $\mathrm{P}$ & 0.29 & -0.33 & 0.30 & 0.29 & 3 \\
\hline & & $\mathrm{Q}$ & 0.25 & -0.25 & 0.25 & 0.25 & 1 \\
\hline & & $\mathrm{R}$ & 0.25 & -0.25 & 0.25 & 0.25 & 1 \\
\hline & & S & 0.30 & -0.30 & 0.30 & 0.30 & 1 \\
\hline & & $\mathrm{T}$ & 0.27 & -0.27 & 0.27 & 0.27 & 1 \\
\hline & & $\mathrm{U}$ & 0.15 & -0.15 & 0.15 & 0.15 & 1 \\
\hline & & $\mathrm{V}$ & 0.23 & -0.23 & 0.23 & 0.23 & 1 \\
\hline & & W & 0.29 & -0.29 & 0.29 & 0.29 & 1 \\
\hline & & $\mathrm{X}$ & 0.28 & -0.28 & 0.28 & 0.28 & 1 \\
\hline & & Average & 0.06 & -0.10 & 0.08 & 0.07 & 5 \\
\hline & Bangladesh & $\mathrm{Y}$ & 0.06 & -0.10 & 0.08 & 0.07 & 5 \\
\hline
\end{tabular}

Table S1. Summary of Arsenic levels in U.S. rice 
Origin Brand

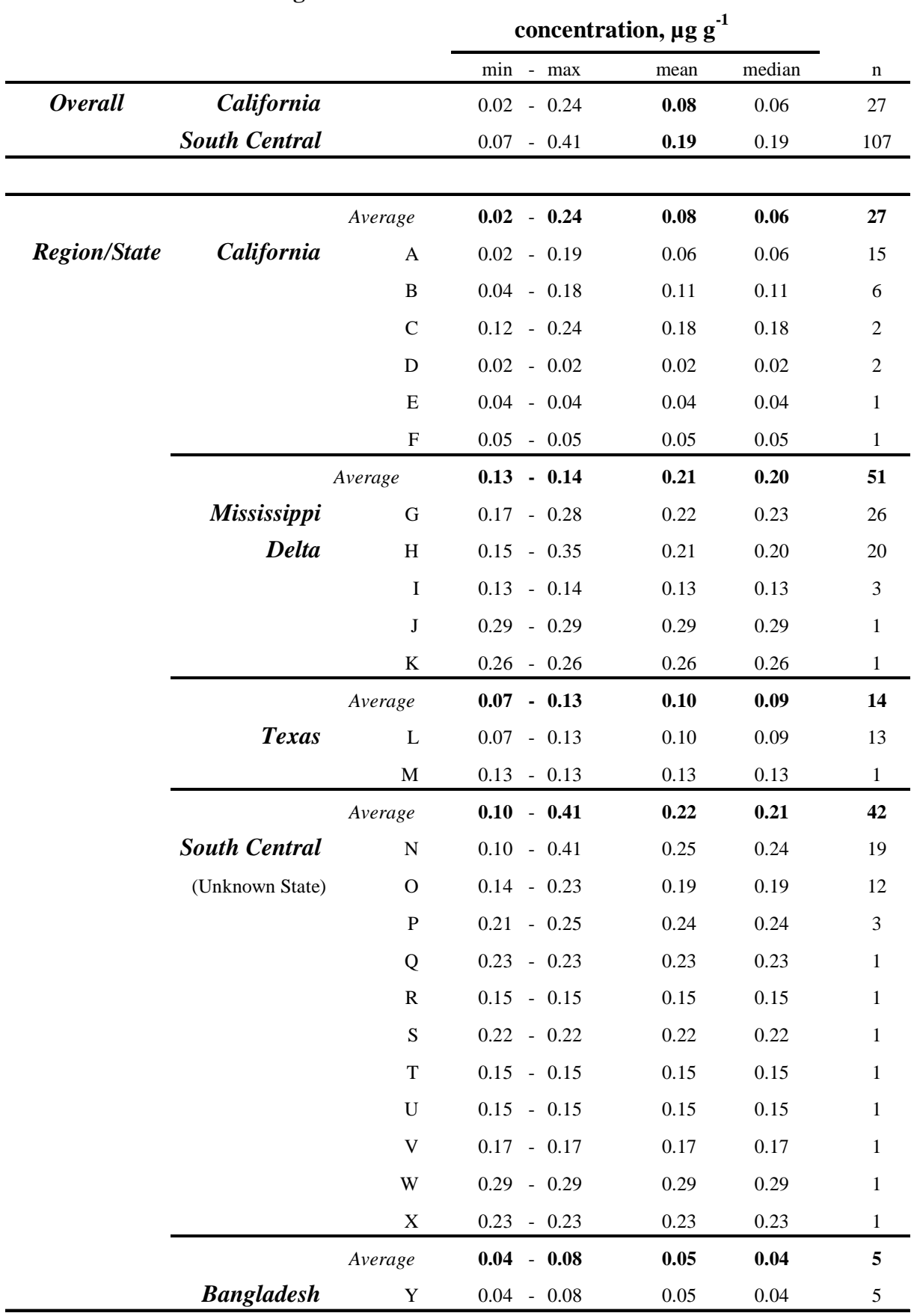

Table S2. Summary of Selenium levels in U.S. rice 


\begin{tabular}{|c|c|c|c|c|c|c|}
\hline & \multirow[t]{2}{*}{ Origin } & \multirow[t]{2}{*}{ Brand } & \multicolumn{3}{|c|}{$\begin{array}{c}\text { Total } \mathrm{Cu} \\
\text { concentration, } \mu \mathrm{g} \mathrm{g}^{-1}\end{array}$} & \multirow[b]{2}{*}{$\mathrm{n}$} \\
\hline & & & $\min -\max$ & mean & median & \\
\hline \multirow[t]{3}{*}{ Overall } & California & & $1.8-5.1$ & 3.1 & 2.9 & 27 \\
\hline & South Central & & $1.6-4.5$ & 2.5 & 2.4 & 107 \\
\hline & & Average & $1.8-5.1$ & 3.1 & 2.9 & 27 \\
\hline \multirow[t]{29}{*}{ Region/State } & California & A & $2.2-5.1$ & 3.5 & 3.5 & 15 \\
\hline & & B & $2.2-2.9$ & 2.7 & 2.8 & 6 \\
\hline & & $\mathrm{C}$ & $2.5-3.0$ & 2.8 & 2.8 & 2 \\
\hline & & $\mathrm{D}$ & $1.8-2.6$ & 2.2 & 2.2 & 2 \\
\hline & & E & $1.8-1.8$ & 1.8 & 1.8 & 1 \\
\hline & & $\mathrm{F}$ & $3.9-3.9$ & 3.9 & 3.9 & 1 \\
\hline & & Average & $1.7-4.5$ & 2.6 & 2.5 & 51 \\
\hline & Mississippi & G & $2.1-3.1$ & 2.5 & 2.4 & 26 \\
\hline & Delta & $\mathrm{H}$ & $1.8-4.5$ & 2.8 & 2.5 & 20 \\
\hline & & I & $1.7-2.2$ & 1.9 & 1.9 & 3 \\
\hline & & $\mathrm{J}$ & $3.5-3.5$ & 3.5 & 3.5 & 1 \\
\hline & & $\mathrm{K}$ & $2.4-2.4$ & 2.4 & 2.4 & 1 \\
\hline & & Average & $1.6-3.0$ & 2.4 & 2.3 & 14 \\
\hline & Texas & $\mathrm{L}$ & $1.8-3.0$ & 2.4 & 2.3 & 13 \\
\hline & & $\mathrm{M}$ & $1.6-1.6$ & 1.6 & 1.6 & 1 \\
\hline & & Average & $1.6-3.9$ & 2.4 & 2.3 & 42 \\
\hline & South Central & $\mathrm{N}$ & $1.9-3.7$ & 2.6 & 2.4 & 19 \\
\hline & (Unknown State) & $\mathrm{O}$ & $1.6-3.9$ & 2.2 & 2.1 & 12 \\
\hline & & $\mathrm{P}$ & $2.0-2.9$ & 2.4 & 2.1 & 3 \\
\hline & & Q & $2.3-2.3$ & 2.3 & 2.3 & 1 \\
\hline & & $\mathrm{R}$ & $2.2-2.2$ & 2.2 & 2.2 & 1 \\
\hline & & $\mathrm{S}$ & $2.8-2.8$ & 2.8 & 2.8 & 1 \\
\hline & & $\mathrm{T}$ & $2.3-2.3$ & 2.3 & 2.3 & 1 \\
\hline & & $\mathrm{U}$ & $1.9-1.9$ & 1.9 & 1.9 & 1 \\
\hline & & $\mathrm{V}$ & $2.4-2.4$ & 2.4 & 2.4 & 1 \\
\hline & & $\mathrm{W}$ & $3.3-3.3$ & 3.3 & 3.3 & 1 \\
\hline & & $\mathrm{X}$ & $2.4-2.4$ & 2.4 & 2.4 & 1 \\
\hline & Bangladesh & Average & $1.6-2.1$ & 1.8 & 1.8 & 5 \\
\hline & & $\mathrm{Y}$ & $1.6-2.1$ & 1.8 & 1.8 & 5 \\
\hline
\end{tabular}

Table S3. Summary of Copper levels in U.S. rice 


\begin{tabular}{|c|c|c|c|c|c|c|c|}
\hline & \multirow[t]{2}{*}{ Origin } & \multirow[t]{2}{*}{ Brand } & \multicolumn{4}{|c|}{$\begin{array}{l}\text { Total Mn } \\
\text { concentration, } \mu \mathrm{g} \mathrm{g}^{-1}\end{array}$} & \multirow[b]{2}{*}{$\mathrm{n}$} \\
\hline & & & $\min$ & $-\max$ & mean & median & \\
\hline \multirow[t]{3}{*}{ Overall } & California & & 7 & -35 & 27 & 19 & 27 \\
\hline & South Central & & 7 & -103 & 16 & 15 & 107 \\
\hline & & Average & 7 & -35 & 27 & 19 & 27 \\
\hline \multirow[t]{29}{*}{ Region/State } & California & A & 14 & -54 & 33 & 37 & 15 \\
\hline & & B & 15 & -38 & 25 & 24 & 6 \\
\hline & & $\mathrm{C}$ & 9 & -10 & 9 & 9 & 2 \\
\hline & & $\mathrm{D}$ & 8 & -9 & 8 & 8 & 2 \\
\hline & & $\mathrm{E}$ & 7 & -7 & 7 & 7 & 1 \\
\hline & & $\mathrm{F}$ & 35 & -35 & 35 & 35 & 1 \\
\hline & & Average & 7 & -103 & 17 & 15 & 51 \\
\hline & Mississippi & G & 7 & -17 & 14 & 14 & 26 \\
\hline & Delta & $\mathrm{H}$ & 11 & -103 & 20 & 16 & 20 \\
\hline & & I & 16 & -17 & 16 & 16 & 3 \\
\hline & & $\mathrm{J}$ & 45 & -45 & 45 & 45 & 1 \\
\hline & & $\mathrm{K}$ & 10 & -10 & 10 & 10 & 1 \\
\hline & & Average & 7 & -32 & 13 & 10 & 14 \\
\hline & Texas & $\mathrm{L}$ & 7 & -32 & 13 & 10 & 13 \\
\hline & & $\mathrm{M}$ & 9 & -9 & 9 & 9 & 1 \\
\hline & & Average & 5 & -46 & 19 & 17 & 42 \\
\hline & South Central & $\mathrm{N}$ & 5 & -46 & 20 & 17 & 19 \\
\hline & (Unknown State) & $\mathrm{O}$ & 9 & -43 & 20 & 14 & 12 \\
\hline & & $\mathrm{P}$ & 8 & -20 & 12 & 10 & 3 \\
\hline & & Q & 16 & -16 & 16 & 16 & 1 \\
\hline & & $\mathrm{R}$ & 15 & -15 & 15 & 15 & 1 \\
\hline & & $S$ & 18 & -18 & 18 & 18 & 1 \\
\hline & & $\mathrm{T}$ & 15 & -15 & 15 & 15 & 1 \\
\hline & & $\mathrm{U}$ & 11 & -11 & 11 & 11 & 1 \\
\hline & & V & 17 & -17 & 17 & 17 & 1 \\
\hline & & W & 43 & -43 & 43 & 43 & 1 \\
\hline & & $\mathrm{X}$ & 17 & -17 & 17 & 17 & 1 \\
\hline & & Average & 17 & -23 & 20 & 20 & 5 \\
\hline & Bangladesh & Y & 17 & -23 & 20 & 20 & 5 \\
\hline
\end{tabular}

Table S4. Summary of Manganese levels in U.S. rice 


\begin{tabular}{|c|c|c|c|c|c|c|c|}
\hline & \multirow[t]{2}{*}{ Origin } & \multirow[t]{2}{*}{ Brand } & \multicolumn{4}{|c|}{$\begin{array}{c}\text { Total Co } \\
\text { concentration, } \mu \mathrm{g} \mathrm{g}^{-1}\end{array}$} & \multirow[b]{2}{*}{$\mathrm{n}$} \\
\hline & & & $\min$ & $-\max$ & mean & median & \\
\hline \multirow[t]{3}{*}{ Overall } & California & & 0.005 & - 0.064 & 0.022 & 0.016 & 27 \\
\hline & South Central & & 0.007 & -0.094 & 0.017 & 0.013 & 107 \\
\hline & & Average & 0.005 & - 0.064 & 0.022 & 0.016 & 27 \\
\hline \multirow[t]{29}{*}{ Region/State } & California & A & 0.012 & - 0.064 & 0.031 & 0.032 & 15 \\
\hline & & B & 0.013 & - 0.019 & 0.015 & 0.016 & 6 \\
\hline & & $\mathrm{C}$ & 0.006 & -0.008 & 0.007 & 0.007 & 2 \\
\hline & & D & 0.005 & -0.009 & 0.007 & 0.007 & 2 \\
\hline & & E & 0.005 & -0.005 & 0.005 & 0.005 & 1 \\
\hline & & $\mathrm{F}$ & 0.016 & -0.016 & 0.016 & 0.016 & 1 \\
\hline & & Average & 0.007 & - 0.056 & 0.015 & 0.013 & 51 \\
\hline & Mississippi & G & 0.011 & - 0.019 & 0.014 & 0.014 & 26 \\
\hline & Delta & $\mathrm{H}$ & 0.007 & - 0.048 & 0.013 & 0.012 & 20 \\
\hline & & I & 0.014 & - 0.017 & 0.016 & 0.016 & 3 \\
\hline & & $\mathrm{J}$ & 0.056 & -0.056 & 0.056 & 0.056 & 1 \\
\hline & & $\mathrm{K}$ & 0.014 & -0.014 & 0.014 & 0.014 & 1 \\
\hline & & Average & 0.007 & - 0.094 & 0.028 & 0.022 & 14 \\
\hline & Texas & $\mathrm{L}$ & 0.007 & - 0.094 & 0.028 & 0.022 & 13 \\
\hline & & M & 0.021 & -0.021 & 0.021 & 0.021 & 1 \\
\hline & & Average & 0.008 & - 0.035 & 0.015 & 0.012 & 42 \\
\hline & South Central & $\mathrm{N}$ & 0.008 & -0.035 & 0.015 & 0.011 & 19 \\
\hline & (Unknown State) & $\mathrm{O}$ & 0.008 & -0.035 & 0.016 & 0.012 & 12 \\
\hline & & $\mathrm{P}$ & 0.011 & -0.018 & 0.014 & 0.013 & 3 \\
\hline & & Q & 0.008 & -0.008 & 0.008 & 0.008 & 1 \\
\hline & & $\mathrm{R}$ & 0.012 & - 0.012 & 0.012 & 0.012 & 1 \\
\hline & & $\mathrm{S}$ & 0.011 & -0.011 & 0.011 & 0.011 & 1 \\
\hline & & $\mathrm{T}$ & 0.014 & - 0.014 & 0.014 & 0.014 & 1 \\
\hline & & $\mathrm{U}$ & 0.012 & -0.012 & 0.012 & 0.012 & 1 \\
\hline & & $\mathrm{V}$ & 0.015 & -0.015 & 0.015 & 0.015 & 1 \\
\hline & & $\mathrm{W}$ & 0.023 & -0.023 & 0.023 & 0.023 & 1 \\
\hline & & $\mathrm{X}$ & 0.016 & -0.016 & 0.016 & 0.016 & 1 \\
\hline & & Average & 0.009 & - 0.014 & 0.011 & 0.010 & 5 \\
\hline & Bangladesh & $\mathrm{Y}$ & 0.009 & -0.014 & 0.011 & 0.010 & 5 \\
\hline
\end{tabular}

Table S5. Summary of Cobalt levels in U.S. rice 


\begin{tabular}{|c|c|c|c|c|c|c|c|}
\hline & \multirow[t]{2}{*}{ Origin } & \multirow[t]{2}{*}{ Brand } & \multicolumn{4}{|c|}{$\begin{array}{c}\text { Total } \mathrm{Zn} \\
\text { concentration, } \mu \mathrm{g} \mathrm{g}^{-1}\end{array}$} & \multirow[b]{2}{*}{$\mathrm{n}$} \\
\hline & & & $\min$ & $-\max$ & mean & median & \\
\hline \multirow[t]{3}{*}{ Overall } & California & & 7 & -42 & 17 & 17 & 27 \\
\hline & South Central & & 9 & -50 & 18 & 17 & 107 \\
\hline & & Average & 7 & -42 & 17 & 17 & 27 \\
\hline \multirow[t]{29}{*}{ Region/State } & California & A & 14 & -22 & 18 & 18 & 15 \\
\hline & & B & 15 & -42 & 21 & 17 & 6 \\
\hline & & $\mathrm{C}$ & 13 & -13 & 13 & 13 & 2 \\
\hline & & D & 8 & -9 & 9 & 9 & 2 \\
\hline & & $\mathrm{E}$ & 7 & -7 & 7 & 7 & 1 \\
\hline & & $\mathrm{F}$ & 18 & -18 & 18 & 18 & 1 \\
\hline & & Average & 9 & -63 & 17 & 16 & 51 \\
\hline & Mississippi & G & 9 & -63 & 15 & 15 & 26 \\
\hline & Delta & $\mathrm{H}$ & 9 & -50 & 19 & 17 & 20 \\
\hline & & I & 15 & -18 & 17 & 18 & 3 \\
\hline & & $\mathrm{J}$ & 32 & -32 & 32 & 32 & 1 \\
\hline & & $\mathrm{K}$ & 10 & -10 & 10 & 10 & 1 \\
\hline & & Average & 13 & -24 & 19 & 19 & 14 \\
\hline & Texas & $\mathrm{L}$ & 13 & -24 & 19 & 19 & 13 \\
\hline & & $\mathrm{M}$ & 23 & -23 & 23 & 23 & 1 \\
\hline & & Average & 8 & -38 & 18 & 18 & 42 \\
\hline & South Central & $\mathrm{N}$ & 8 & -38 & 18 & 16 & 19 \\
\hline & (Unknown State) & $\mathrm{O}$ & 14 & -32 & 19 & 17 & 12 \\
\hline & & $\mathrm{P}$ & 10 & -26 & 18 & 18 & 3 \\
\hline & & Q & 15 & -15 & 15 & 15 & 1 \\
\hline & & $\mathrm{R}$ & 19 & -19 & 19 & 19 & 1 \\
\hline & & $\mathrm{S}$ & 18 & -18 & 18 & 18 & 1 \\
\hline & & $\mathrm{T}$ & 19 & -19 & 19 & 19 & 1 \\
\hline & & $\mathrm{U}$ & 21 & -21 & 21 & 21 & 1 \\
\hline & & $\mathrm{V}$ & 19 & -19 & 19 & 19 & 1 \\
\hline & & $\mathrm{W}$ & 23 & -23 & 23 & 23 & 1 \\
\hline & & $\mathrm{X}$ & 18 & -18 & 18 & 18 & 1 \\
\hline & & Average & 11 & -14 & 12 & 12 & 5 \\
\hline & Bangladesh & $\mathrm{Y}$ & 11 & -14 & 12 & 12 & 5 \\
\hline
\end{tabular}

Table S6. Summary of Zinc levels in U.S. rice 


\section{Brand Code}

$\begin{array}{rc}\text { Lundberg } & \text { A } \\ \text { Hinode/Pacific Basin Rice Products } & \mathrm{B} \\ \text { Koda Farms } & \mathrm{C} \\ \text { Nishiki } & \mathrm{D} \\ \text { Unknown } & \mathrm{E} \\ \text { Nijiya Market/Torrance } & \mathrm{F} \\ \text { Producers Rice } & \mathrm{G} \\ \text { Riceland } & \mathrm{H} \\ \text { Cajun Country/Falcon Rice Mill } & \mathrm{I} \\ \text { Konriks/Conrad Rice Mill } & \mathrm{J} \\ \text { Zatarain's } & \mathrm{K} \\ \text { Rice Select } & \mathrm{L} \\ \text { Lotus Foods } & \mathrm{M} \\ \text { Water Maid/Riviana } & \mathrm{N} \\ \text { Shurfine } & \mathrm{O} \\ \text { Great Value/Wal-Mart } & \mathrm{P} \\ \text { Always Save/Associated Wholesame Grocers Inc } & \mathrm{Q} \\ \text { American Value/Dolgencarp } & \mathrm{R} \\ \text { Best Choice/Associated Wholesame Grocers Inc } & \mathrm{S} \\ \text { Rice Town/Save-a-lot } & \mathrm{T} \\ \text { Clover Valley/Dolgencarp } & \mathrm{U} \\ \text { Flavorite/ Supervada } & \mathrm{V} \\ \text { Kroger } & \mathrm{W} \\ \text { Vitarroz } & \mathrm{X}\end{array}$

Table S7. US Rice Brands. 


\begin{tabular}{|c|c|c|c|c|c|c|c|c|}
\hline \multirow[t]{2}{*}{ origin } & \multirow[t]{2}{*}{ treatment } & \multicolumn{6}{|c|}{ mean total concentration, $\mu \mathrm{g} \mathrm{g}^{-1}$} & \multirow[t]{2}{*}{$\mathbf{n}$} \\
\hline & & Co & Se & As & $\mathbf{C u}$ & $\mathbf{Z n}$ & Mn & \\
\hline & Unpolished & 0.034 & 0.11 & 0.25 & 3.4 & 22 & 44 & 22 \\
\hline & Polished & 0.015 & 0.19 & 0.28 & 2.5 & 17 & 14 & 112 \\
\hline \multirow[t]{2}{*}{ California } & Unpolished & 0.032 & 0.05 & 0.17 & 3.4 & 20 & 42 & 13 \\
\hline & Polished & 0.013 & 0.10 & 0.17 & 2.8 & 15 & 13 & 14 \\
\hline \multirow{2}{*}{$\begin{array}{l}\text { South } \\
\text { Central }\end{array}$} & Unpolished & 0.037 & 0.19 & 0.37 & 3.5 & 24 & 47 & 9 \\
\hline & Polished & 0.015 & 0.20 & 0.29 & 2.4 & 17 & 15 & 98 \\
\hline
\end{tabular}

Table S8. Summary of the element levels in polished/unpolished US Rice. 


\begin{tabular}{rcccc} 
& \multicolumn{5}{c}{ Manganese } \\
& $\begin{array}{c}\text { Degrees } \\
\text { freedom }\end{array}$ & $\begin{array}{c}\text { adjusted } \\
\text { means square }\end{array}$ & $\mathrm{F}$ & $\mathrm{P}$ \\
California/South Central & 1 & 74 & 0.08 & 0.777 \\
$\begin{array}{c}\text { Polished/ } \\
\text { Unpolished }\end{array}$ & 1 & 68272 & 73.9 & $<0.001$ \\
$\begin{array}{c}\text { California/South Central } \\
* \text { Polished/Unpolished }\end{array}$ & 1 & 128 & 0.14 & 0.71 \\
error & 130 & 924 & &
\end{tabular}

\section{Cobalt}

$\begin{array}{rcccc}\text { California/South Central } & 1 & 479 & 0.44 & 0.509 \\ \begin{array}{r}\text { Polished/ } \\ \text { Unpolished }\end{array} & 1 & 50998 & 46.6 & <0.001 \\ \begin{array}{r}\text { California/South Central } \\ * \text { Polished/Unpolished }\end{array} & 1 & 1 & 0.00 & 0.974 \\ \text { error } & 130 & 1093 & & \end{array}$

\section{Copper}

$\begin{array}{rcccc}\text { California/South Central } & 1 & 1090 & 1.01 & 0.317 \\ \begin{array}{r}\text { Polished/ } \\ \text { Unpolished }\end{array} & 1 & 35745 & 33 & <0.001 \\ \begin{array}{r}\text { California/South Central } \\ * \text { Polished/Unpolished }\end{array} & 1 & 954 & 0.88 & 0.35 \\ \text { error } & 130 & 1083 & & \end{array}$

Table S9. Analysis of variance for transition metals. Data ranked prior to analysis to normalise the distribution. 


\begin{tabular}{rcccc} 
& \multicolumn{5}{c}{ Arsenic } \\
& $\begin{array}{c}\text { Degrees } \\
\text { freedom }\end{array}$ & $\begin{array}{c}\text { adjusted } \\
\text { means square }\end{array}$ & $\mathrm{F}$ & $\mathrm{P}$ \\
California/South Central & 1 & 57118 & 53.41 & $<0.001$ \\
Polished/ & 1 & 1673 & 1.56 & 0.213 \\
$\begin{array}{r}\text { Unpolished } \\
\text { California/South Central }\end{array}$ & 1 & 4242 & 3.97 & 0.049 \\
errolished/Unpolished & 130 & 1069 & &
\end{tabular}

\section{Selenium}

$\begin{array}{rcccc}\text { California/South Central } & 1 & 44026 & 43.78 & <0.001 \\ \begin{array}{r}\text { Polished/ } \\ \text { Unpolished }\end{array} & 1 & 2557 & 2.54 & 0.113 \\ \begin{array}{r}\text { Californai/South Central } \\ * \text { Polished/Unpolished }\end{array} & 1 & 1480 & 1.47 & 0.227 \\ \text { error } & 130 & 1006 & & \end{array}$

Table S10. Analysis of variance for arsenic and selenium. Data ranked prior to analysis to normalise the distribution 


\section{Transition Metals}
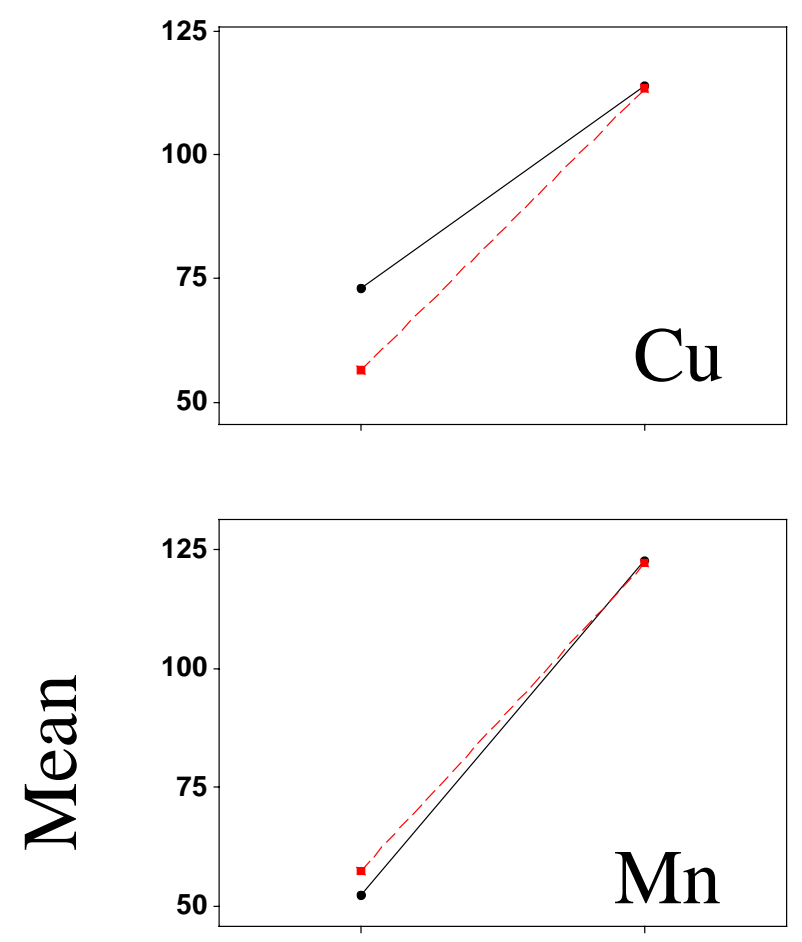

\section{Semi-Metals}
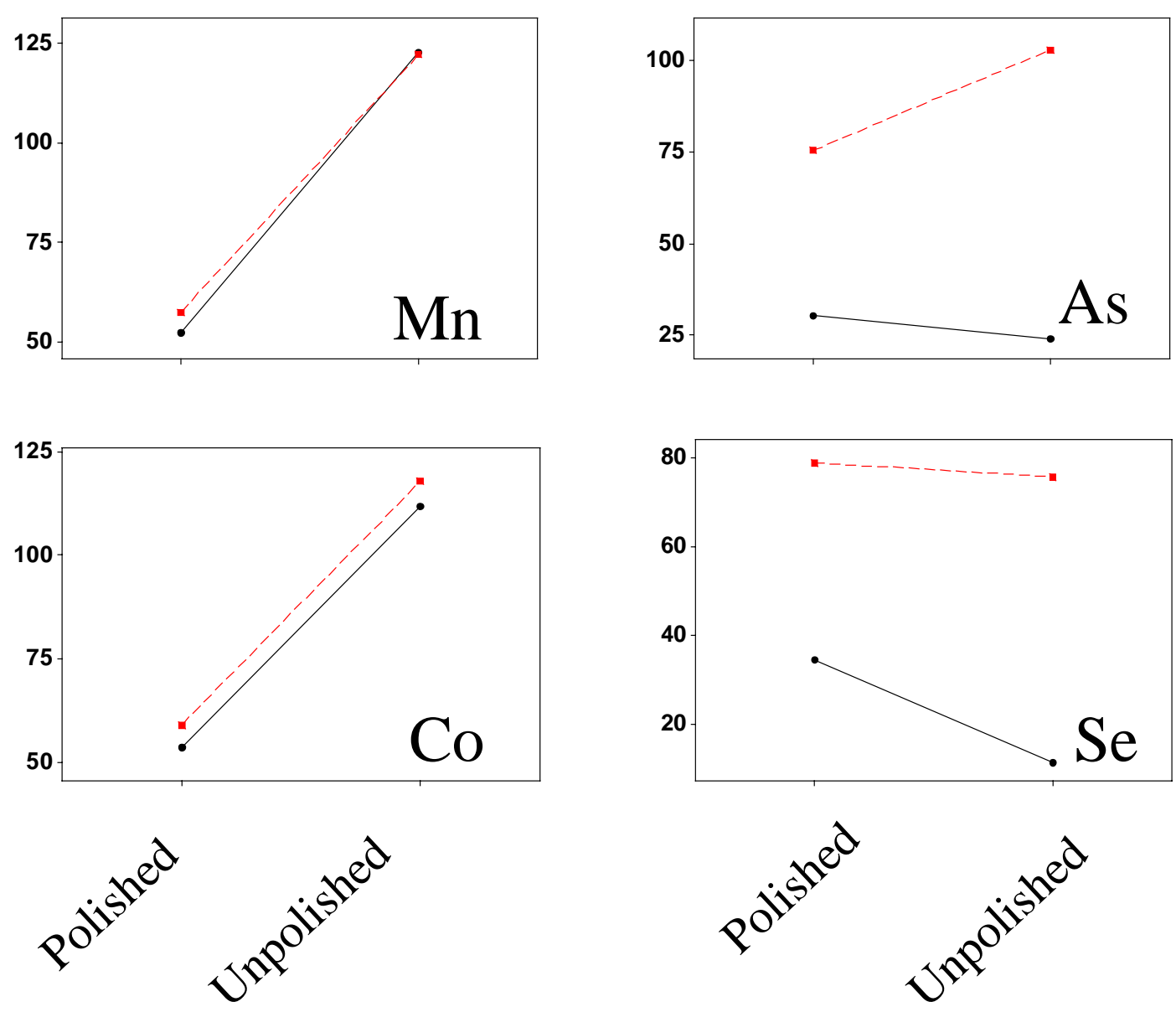

Fig S1. Interaction plots for polished and unpolished rice from either

Californian or South Central US rice. Solid line $=$ Californian rice. Dashed line $=$ South Central rice 


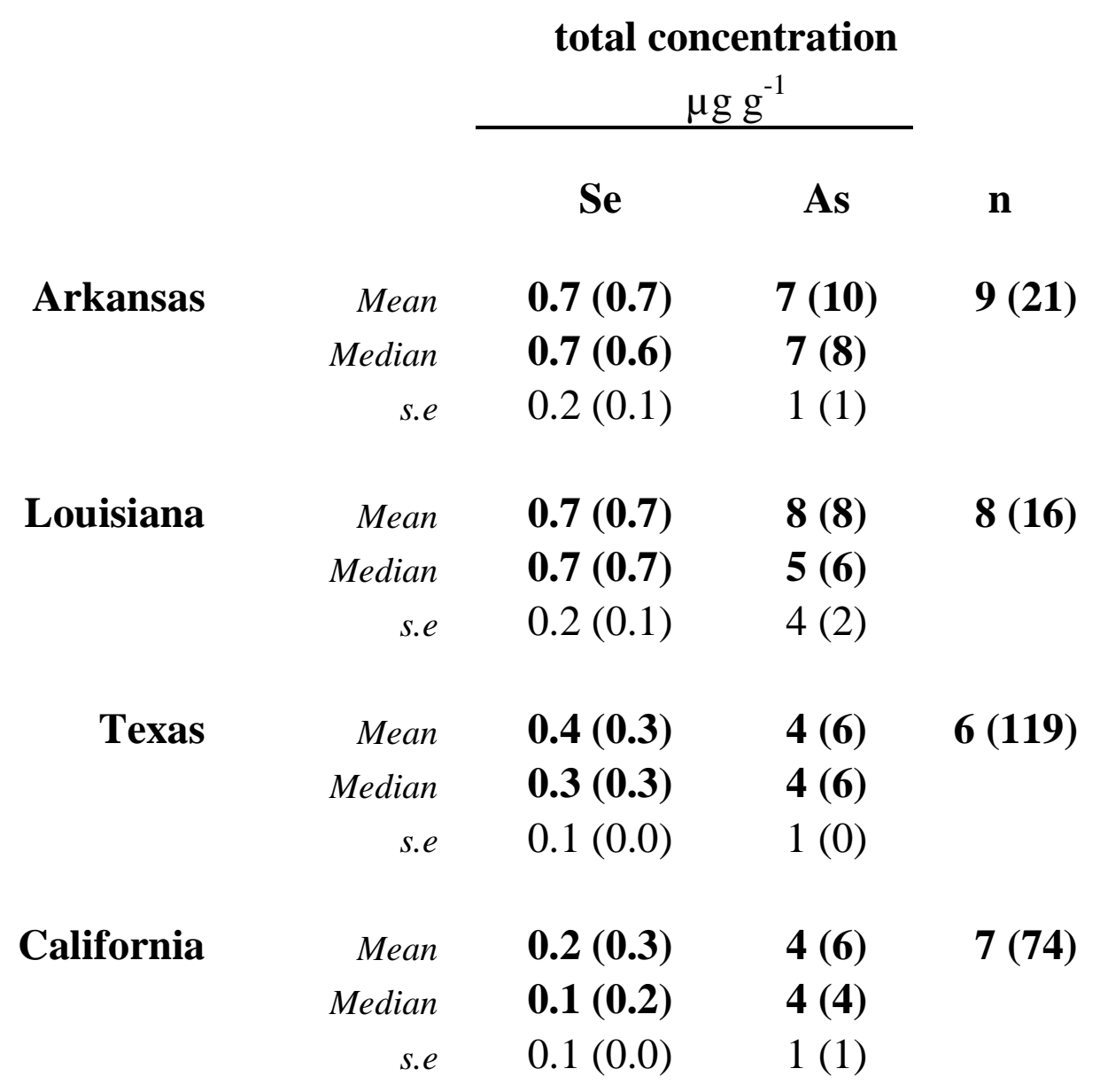

Table S11. Background levels of As and Se in soils from non-agricultural soils from rice producing regions of the US. Levels taken from the Shacklette database (1). 


$\begin{array}{rcc}\begin{array}{r}\text { Mississippi soils } \\ \text { by region }\end{array} & \begin{array}{c}\text { Mean } \\ \text { arsenic } \\ \text { concentrations } \\ \text { Mg As } \mathbf{~ g}^{-1}\end{array} & \mathbf{n} \\ & & \\ \text { Delta* } & 15.02 & 31 \\ \text { Interior Flatwoods } & 12.73 & 7 \\ \text { Blackland Prairie } & 11.11 & 24 \\ \text { Loess } & 9.31 & 42 \\ \text { Upper Coastal Plain } & 6.58 & 61 \\ \text { Lower Coastal Plain } & 5.79 & 81 \\ \text { Coastal Flatwoods } & 4.42 & 14\end{array}$

$\begin{array}{rcc}\begin{array}{c}\text { Mississippi Soil } \\ \text { by surface } \\ \text { horizon }\end{array} & \begin{array}{c}\text { Mean } \\ \text { arsenic } \\ \text { concentrations } \\ \text { in soil solution } \\ \mathbf{\mu g ~ A s ~ g ~}^{-1}\end{array} & \mathbf{n} \\ \text { Undisturbed } & 3.63 & 39 \\ \text { Cultivated } & 8.09 & 90\end{array}$

Table S12. Summary of total and water soluble As in soils from the State of Mississippi. Significant differences $(p<0.05)$ between undisturbed/cultivated mean As soil solution levels. Significant differences $(\mathrm{p}<0.05)$ in mean soil As levels between Delta and Blackland Praire, Loess, Upper Coastal Plain, Lower Coastal Plain and Coastal Flatwoods regions. Data from Pettry \& Switzer (2). 
*indicates regions of rice production

Mean Concentration $\pm \operatorname{s.e}\left(\mu \mathrm{g} \mathrm{g}^{-1}\right)$

As

$\begin{array}{rr}\text { Mississippi } & \text { Arkansas } \\ \text { Delta } & \text { Louisiana } \\ & \text { Mississippi } \\ & \text { Missouri }\end{array}$

Gulf of Texas
Texas

$1.40 \pm 0.12$

$1.62 \pm 0.08$

$1.29 \pm 0.10$

$2.25 \pm 0.12$

$2.11 \pm 0.17$

$0.24 \pm 0.02$

$\mathbf{0 . 3 4} \pm 0.02$

$\mathbf{0 . 3 0} \pm 0.02$

$\mathbf{0 . 5 5} \pm 0.04$

$\mathbf{0 . 4 2} \pm 0.09$

California

California

$4.27 \pm 0.23$

$\mathbf{0 . 2 2} \pm 0.02$

13

Table S13. Summary of As and Se levels in stream sediments from rice producing counties in the US. Significant differences between the Mississippi Delta, Gulf of Texas and California stream sediments in areas used to produce rice were observed for As (GLM, p < 0.001) and Se (GLM, p < 0.001). Data from USGS Geochemical survey (3). 


\begin{tabular}{|c|c|c|c|c|}
\hline & \multicolumn{3}{|c|}{ Potable groundwater As level $\mu \mathrm{g} \mathrm{g}^{-1}$} & \multirow[b]{2}{*}{$\mathbf{n}$} \\
\hline & Min - Max & Mean & Median & \\
\hline Arkansas & $<1-80$ & $3 \pm 0.5$ & 1 & 216 \\
\hline Louisiana & $<1-200$ & $2 \pm 0.7$ & 1 & 299 \\
\hline Texas & $<1-77$ & $2 \pm 0.2$ & 1 & 884 \\
\hline California & $<1-2600$ & $12 \pm 1.5$ & 2 & 2180 \\
\hline
\end{tabular}

Table S14. Summary of As levels in potable groundwaters, by State. Data from USGS National Water Information System 2001 (4). 


\begin{tabular}{|c|c|c|c|c|c|c|c|}
\hline $\begin{array}{l}\text { Isotope } \\
\text { Element }\end{array}$ & & $\begin{array}{l}59 \\
\text { Co }\end{array}$ & $\begin{array}{l}78 \\
\mathbf{S e}\end{array}$ & $\begin{array}{l}75 \\
\text { As }\end{array}$ & $\begin{array}{l}63 \\
\mathrm{Cu}\end{array}$ & $\begin{array}{l}64 \\
\mathbf{Z n}\end{array}$ & $\begin{array}{c}55 \\
\mathbf{M n}\end{array}$ \\
\hline \multirow[t]{8}{*}{ Blank } & 1 & 0.008 & 0.06 & 0.05 & 0.8 & 0 & 1 \\
\hline & 2 & 0.008 & 0.06 & 0.07 & 0.3 & 6 & 1 \\
\hline & 3 & 0.009 & 0.07 & 0.06 & 1.3 & 2 & 1 \\
\hline & 4 & 0.014 & 0.06 & 0.06 & 1.2 & 4 & 1 \\
\hline & 5 & 0.008 & 0.07 & 0.05 & 0.7 & 1 & 1 \\
\hline & 6 & 0.009 & 0.07 & 0.05 & 0.5 & 5 & 1 \\
\hline & 7 & 0.009 & 0.07 & 0.05 & 0.5 & 2 & 1 \\
\hline & 8 & 0.008 & 0.06 & 0.05 & 0.4 & 1 & 1 \\
\hline Mean & $\mu g \mathrm{~L}^{-1}$ & 0.009 & 0.06 & 0.05 & 0.7 & 3 & 1 \\
\hline Limits of & $\mu \mathrm{g} \mathrm{L}^{-1}$ & 0.015 & 0.079 & 0.077 & 1.790 & 8.763 & 1.348 \\
\hline Detection & $\mu \mathrm{g} \mathrm{g}^{-1 *}$ & 0.004 & 0.020 & 0.019 & 0.448 & 2.191 & 0.337 \\
\hline \multirow[t]{4}{*}{ Spike } & 1 & 1.094 & 3.60 & 4.49 & 27 & 38 & 54 \\
\hline & 2 & 1.045 & 3.95 & 4.61 & 25 & 42 & 54 \\
\hline & 3 & 1.050 & 3.95 & 4.62 & 25 & 40 & 54 \\
\hline & 4 & 1.009 & 3.70 & 4.29 & 24 & 36 & 51 \\
\hline Mean & $\mu \mathrm{g} \mathrm{L}^{-1}$ & 1 & 4 & 5 & 25 & 39 & 54 \\
\hline Spike value & $\mu \mathrm{g} \mathrm{L}^{-1}$ & 1 & 5 & 5 & 25 & 50 & 50 \\
\hline Recovery & $\%$ & 105 & 76 & 90 & 102 & 78 & 107 \\
\hline \multirow[t]{7}{*}{ CRM } & 1 & 0.016 & 0.35 & 0.32 & 2.51 & 15 & 19 \\
\hline & 2 & 0.016 & 0.36 & 0.33 & 2.39 & 15 & 19 \\
\hline & 3 & 0.016 & 0.38 & 0.33 & 2.35 & 17 & 20 \\
\hline & 4 & 0.017 & 0.37 & 0.32 & 2.23 & 15 & 19 \\
\hline & 5 & 0.016 & 0.38 & 0.34 & 2.29 & 16 & 19 \\
\hline & 6 & 0.014 & 0.36 & 0.31 & 2.09 & 14 & 18 \\
\hline & 7 & 0.016 & 0.37 & 0.33 & 2.27 & 16 & 19 \\
\hline Mean & $\mu \mathrm{g} \mathrm{g}^{-1}$ & 0.016 & 0.37 & 0.33 & 2.3 & 15 & 18 \\
\hline Certified value & $\mu \mathrm{g} \mathrm{g}^{-1}$ & 0.018 & 0.38 & 0.29 & 2.4 & 19.4 & 20 \\
\hline Recovery & $\%$ & 88 & 96 & 112 & 97 & 77 & 91 \\
\hline
\end{tabular}

Table S15. Quality Control. Method blanks, spikes and certified reference material. * Assuming sample weights of $0.2 \mathrm{~g}$ are maintained 
Total Grain Level, $\mu \mathrm{g}$ g-1 d.wt

\begin{tabular}{|c|c|c|c|c|c|c|c|c|c|c|c|}
\hline & 59 & 78 & 75 & 63 & 64 & 55 & Brand & Description & Date Bought & Shop Location & Shop Bought \\
\hline & Co & Se & As & $\mathbf{C u}$ & Zn & Mn & & & & & \\
\hline Average & 0.032 & 0.07 & 0.16 & 3.9 & 20 & 44 & Lundberg & Brown & Friday 23rd Sept & Sacromento & Organic CO-OP \\
\hline \multirow[t]{2}{*}{ s.e. } & 0.000 & 0.00 & 0.00 & 0.2 & 0 & 1 & & Basmati & & & \\
\hline & 0 & 7 & 2 & 11 & 5 & 2 & & Organic & & & \\
\hline Average & 0.017 & 0.04 & 0.25 & 2.8 & 28 & 36 & Hinode & Brown & Monday 26th Sept & Sacromento & Safeway \\
\hline \multirow[t]{2}{*}{ s.e. } & 0.002 & 0.00 & 0.03 & 0.1 & 14 & 2 & & Long Grain & & & \\
\hline & 20 & 11 & 26 & 6 & 98 & 3 & & & & & \\
\hline Average & 0.009 & 0.08 & 0.09 & 1.6 & 11 & 17 & Lotus Foods & Baby Basmati & Friday 23rd Sept & Sacromento & Organic CO-OP \\
\hline \multirow[t]{2}{*}{ s.e. } & 0.000 & 0.00 & 0.00 & 0.0 & 1 & 0 & & imported from & & & \\
\hline & 6 & 3 & 5 & 1 & 12 & 5 & & Bangladesh & & & \\
\hline Average & 0.012 & 0.19 & 0.25 & 2.4 & 19 & 15 & Riceland & White & Saturday 17th Sept & Little Rock & Harvest Foods \\
\hline \multirow[t]{2}{*}{ s.e. } & 0.000 & 0.01 & 0.01 & 0.1 & 2 & 1 & & Extra Long Grain & & & \\
\hline & 3 & 6 & 11 & 9 & 19 & 7 & & Enriched & & & \\
\hline Average & 0.014 & 0.25 & 0.27 & 3.0 & 17 & 17 & Produces & White & Monday 19th Sept & Stuttgart & Walmart \\
\hline \multirow[t]{2}{*}{ s.e. } & 0.001 & 0.01 & 0.01 & 0.2 & 1 & 0 & & Long Grain & & & \\
\hline & 12 & 6 & 5 & 16 & 6 & 5 & & Enriched & & & \\
\hline Average & 0.013 & 0.19 & 0.27 & 2.5 & 17 & 15 & Producers & White & Monday 19th Sept & Stuttgart & Mayflower \\
\hline \multirow[t]{2}{*}{ s.e. } & 0.001 & 0.01 & 0.00 & 0.2 & 0 & 1 & & Long Grain & & & \\
\hline & 14 & 8 & 2 & 16 & 6 & 7 & & Enriched & & & \\
\hline Average & 0.012 & 0.16 & 0.23 & 2.3 & 18 & 16 & Riceland & White & Monday 19th Sept & Stuttgart & Mayflower \\
\hline \multirow[t]{2}{*}{ s.e. } & 0.001 & 0.00 & 0.00 & 0.2 & 1 & 0 & & Extra Long Grain & & & \\
\hline & 8 & 1 & 1 & 16 & 14 & 6 & & Enriched & & & \\
\hline Average & 0.020 & 0.18 & 0.28 & 3.0 & 16 & 13 & Par Excellence & Premium Long Grain & Monday 19th Sept & Stuttgart & Mayflower \\
\hline \multirow[t]{2}{*}{ s.e. } & 0.003 & 0.00 & 0.01 & 0.4 & 6 & 1 & & Parboiled & & & \\
\hline & 32 & 2 & 8 & 27 & 75 & 12 & & Enriched & & & \\
\hline Average & 0.010 & 0.29 & 0.26 & 2.3 & 31 & 19 & Mahatma & White & Monday 19th Sept & Stuttgart & Mayflower \\
\hline \multirow[t]{2}{*}{ s.e. } & 0.000 & 0.00 & 0.00 & 0.0 & 8 & 0 & & Extra Long Grain & & & \\
\hline & 8 & 0 & 3 & 0 & 50 & 1 & & Enriched & & & \\
\hline Average & 0.023 & 0.08 & 0.35 & 1.9 & 22 & 13 & Rice Select & Texmati & Thursday 15 th Sept & Little Rock & Kroger \\
\hline \multirow[t]{2}{*}{ s.e. } & 0.001 & 0.00 & 0.01 & 0.0 & 7 & 0 & & & & & \\
\hline & 6 & 7 & 8 & 4 & 64 & 86 & & & & & \\
\hline Average & 0.010 & 0.20 & 0.25 & 1.9 & 15 & 20 & Water Maid & White & Friday 16th Sept & Little Rock & Harvest Foods \\
\hline \multirow[t]{2}{*}{ s.e. } & 0.001 & 0.01 & 0.01 & 0.1 & 1 & 1 & & Medium Grain & & & \\
\hline & 16 & 10 & 11 & 16 & 13 & 15 & & Enriched & & & \\
\hline Average & 0.013 & 0.16 & 0.26 & 2.3 & 19 & 16 & American Value & White & Friday 16th Sept & Little Rock & Dollar General \\
\hline \multirow[t]{2}{*}{ s.e. } & 0.001 & 0.01 & 0.01 & 0.2 & 0 & 1 & & Extra Long Grain & & & \\
\hline & 13 & 10 & 5 & 31 & 4 & 10 & & Enriched & & & \\
\hline Average & 0.032 & 0.16 & 0.28 & 2.2 & 20 & 19 & Price Saver & White & Friday 16th Sept & Little Rock & Harvest Foods \\
\hline \multirow[t]{2}{*}{ s.e. } & 0.001 & 0.00 & 0.00 & 0.0 & 0 & 0 & & Long Grain & & & \\
\hline & 4 & 5 & 1 & 3 & 5 & 2 & & Enriched & & & \\
\hline Average & 0.016 & 0.23 & 0.28 & 2.3 & 18 & 16 & Vitarroz & White & Thursday 15 th Sept & Little Rock & Alps Market \\
\hline \multirow[t]{2}{*}{ s.e. } & 0.000 & 0.00 & 0.01 & 0.2 & 1 & 1 & & Extra Long Grain & & & \\
\hline & 5 & 2 & 4 & 16 & 6 & 9 & & Enriched & & & \\
\hline Average & 10 & 6 & 7 & 12 & 27 & 12 & & & & & \\
\hline s.e & 2 & 1 & 2 & 2 & 8 & 6 & & & & & \\
\hline
\end{tabular}

Table S16. Duplicate analysis of samples. Relative Difference $=(($ rep a - rep b)/mean of rep a \& b) )*100. 


\begin{tabular}{c|cccccc} 
& \multicolumn{6}{c}{ concentration, $\boldsymbol{\mu g} \mathbf{L}^{-2}$} \\
Standard & $\mathbf{C o}$ & $\mathbf{S e}$ & $\mathbf{A s}$ & $\mathbf{C u}$ & $\mathbf{Z n}$ & $\mathbf{M n}$ \\
\hline $\mathbf{1}$ & 0 & 0 & 0 & 0 & 0 & 0 \\
$\mathbf{3}$ & 0.05 & 0.25 & 0.25 & 1.25 & 2.5 & 2.5 \\
$\mathbf{4}$ & 0.2 & 1 & 1 & 5 & 10 & 10 \\
$\mathbf{5}$ & 0.4 & 2 & 2 & 10 & 20 & 20 \\
& 2 & 10 & 10 & 50 & 100 & 100
\end{tabular}

Table S17. Composition and concentration of mulielement standard. 

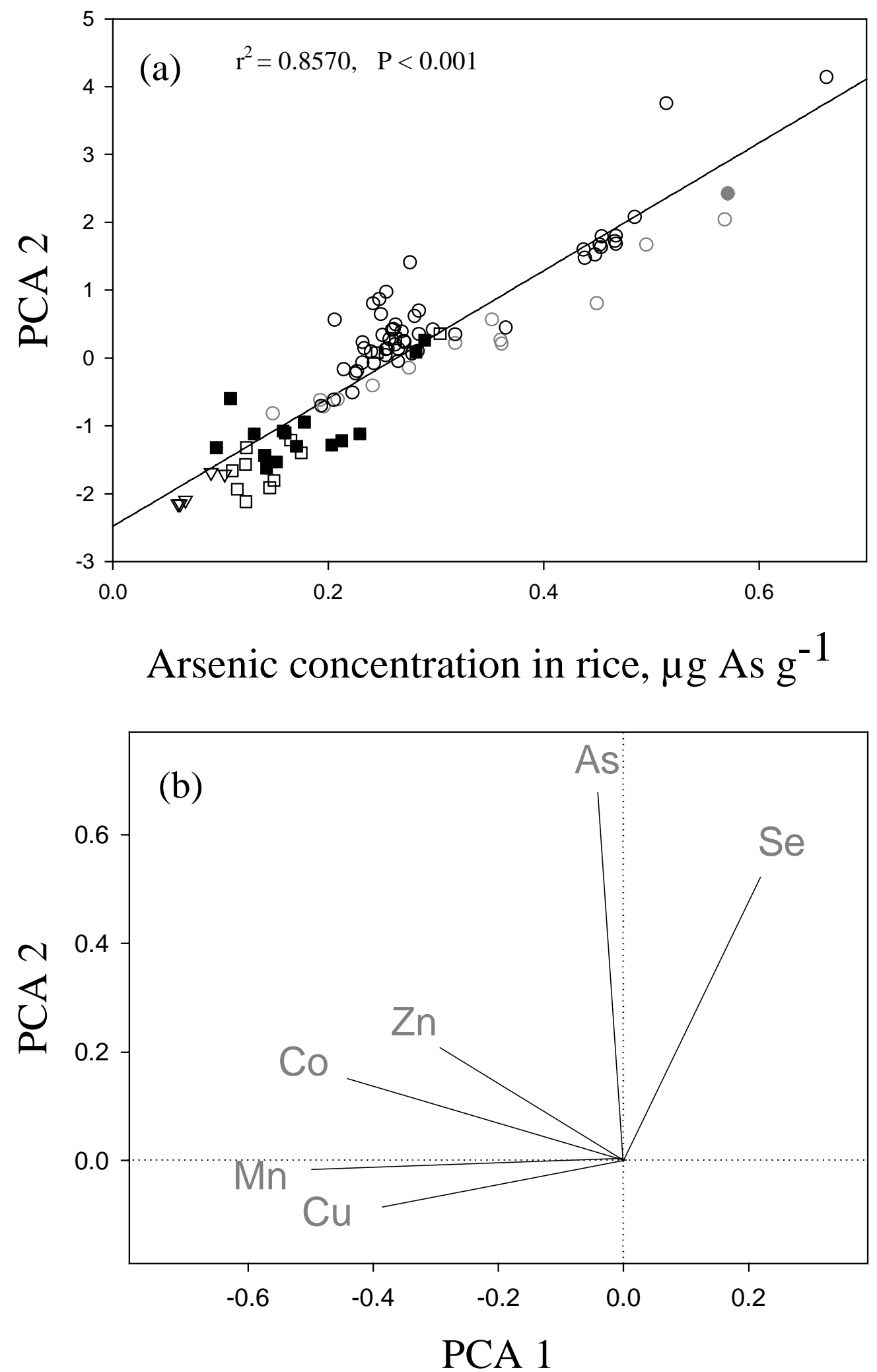

Fig S2. (a) Regression of As grain level vs. PCA Axis 2. Circles = South Central USA (Black: Mississippi Delta [ $n=51]$; Grey $=$ Gulf Coast of Texas [ $n=14]$ ). Black Square $=$ California $[n=27]$. Triangle $=$ Bangladesh $[n=5]$. Filled Symbols indicate unpolished rice. (b) Loading plot of As, Se, Cu, Mn, Co, Zn. 


\section{References}

(1) United States Geological Survey (USGS),. http://tin.er.usgs.gov/ussoils, 2006; (June,2006).

(2) Pettry, D.E.; Switzer, R.E. Arsenic concentrations in selected soils and parent materials in Mississippi, MAFFS Bulletin 1104. 2001, Office of Agriculture Communications, Mississippi State University.

(3) USGS National Geochemical Survey. http://tin.er.usgs.gov/geochem/doc/home.htm, 2006; (April, 2006).

(4) USGS National Water Information System. http://water.usgs.gov/nawqa/trace/data/arsenic nov2001.txt, 2001; (April, 2006). 